\title{
SCHUR'S THEOREM FOR MODIFIED DISCRETE FOURIER TRANSFORM
}

\author{
N. O. Kotelina And A. B. Pevnyi
}

Abstract. We find the eigenvalues of modified Fourier matrix $S$ with entries $S_{k j}=\frac{1}{\sqrt{n}} \omega^{k(1-j)}$, $0 \leqslant k, j \leqslant n-1$, where $\omega=\exp \frac{2 \pi i}{n}$. For this matrix $S^{4}=\omega I$. The matrix has an interesting property: for $n=4 m$ eigenvalues have equal multiplicities. We prove a theorem giving the multiplicities of eigenvalues for all $n$. The theorem is similar to Schur's theorem (1921) for standard Fourier matrix. Our proofs are self-contained. In the proof we calculate modified Gauss sums by means of the classical analysis.

Mathematics subject classification (2010): 42A99.

Keywords and phrases: Schur's theorem, discrete Fourier transform, modified discrete Fourier transform, eigenvalues.

\section{REFERENCES}

[1] B. C. Berndt, R. J. Evans And K. S. Williams, Gauss and Jacobi sums, 598 pp. Wiley, New York, 1998.

[2] I. SCHUR, Über die Gaussschen summen, Nach. Gessel. Göttingen, Math-Phys Klasse, pp. 147-153 (1921).

[3] S. M. SitniK, Modified discrete Fourier transform, [in Russian]. Vestnik of Voronezh institute of MVD of Russia. 36, 7 (2006), 196-201. 